University of Nebraska - Lincoln

DigitalCommons@University of Nebraska - Lincoln

2005

\title{
Role of Branching on the Structure of Polymer Brushes Formed from Comb Copolymers
}

\author{
Peng Tian \\ David Uhrig \\ Jimmy W. Mays \\ Hiroshi Watanabe \\ S. Michael Kilbey, II
}

Follow this and additional works at: https://digitalcommons.unl.edu/usdoepub

Part of the Bioresource and Agricultural Engineering Commons

Tian, Peng; Uhrig, David; Mays, Jimmy W.; Watanabe, Hiroshi; and Kilbey, II, S. Michael, "Role of Branching on the Structure of Polymer Brushes Formed from Comb Copolymers" (2005). US Department of Energy Publications. 57.

https://digitalcommons.unl.edu/usdoepub/57

This Article is brought to you for free and open access by the U.S. Department of Energy at DigitalCommons@University of Nebraska - Lincoln. It has been accepted for inclusion in US Department of Energy Publications by an authorized administrator of DigitalCommons@University of Nebraska - Lincoln. 


\section{Notes}

\section{Role of Branching on the Structure of Polymer Brushes Formed from Comb Copolymers}

\author{
Peng Tian, ${ }^{\dagger}$ David Uhrig, ${ }^{\dagger}$ Jimmy W. Mays, ${ }^{\ddagger}$, \\ Hiroshi Watanabe," and S. Michael Kilbey, II*,† \\ Department of Chemical Engineering, Clemson University, \\ Clemson, South Carolina 29634, Chemical Sciences \\ Division, Oak Ridge National Laboratory, \\ Oak Ridge, Tennessee 37831, Department of Chemistry, \\ University of Tennessee, Knoxville, Tennessee 37996, \\ Institute for Chemical Research, Kyoto University, Uji, \\ Kyoto 611-0011, Japan \\ Received May 18, 2004 \\ Revised Manuscript Received December 28, 2004
}

The distinguishing feature of a polymer brush at equilibrium is the stretched configuration of the chains that results from tethering the polymer chains by one end at the solid-fluid interface. ${ }^{1-5}$ The stretched configuration of the chains and the crowded nature of the interfacial layer is the origin of many of the useful properties of polymer brushes: these layers resist compression and aggregation, effectively dissipate shear stresses, and respond reversibly to changes in their solution environment.

One of the most useful methods of brush formation for fundamental studies is preferentially adsorbing rigorously synthesized, amphiphilic block copolymers to a surface. ${ }^{1-3}$ The structure of brushes created by preferential adsorption of linear polymer amphiphiles can be adequately predicted by single-chain, mean-field models. ${ }^{4,5}$ These models are extremely useful because they predict average thermodynamic properties and scaling dependencies, despite ignoring local features. In this Note, we report on the structure and scaling of linear polymer brushes created by preferentially adsorbed, branched polymer amphiphiles. As revealed by the scaling analysis, the structure of brushes produced from these materials is significantly impacted by local details-namely, the connectivity of the macromolecule.

We have used a surface forces apparatus (SFA) and procedures well described for brushes formed from diblock copolymers ${ }^{2,3}$ to measure the normal forces as a function of surface separation distance between opposing brushes made from comb copolymers. The regular multigraft combs consist of polyisoprene (PI) backbones with polystyrene (PS) grafts, and the synthesis and molecular weight characterization of these materials has been previously reported. ${ }^{6}$ The three combs used are identified as follows 3.3[48/60], 3.5[34/77], and 3.5-

\footnotetext{
* Author to whom correspondence should be addressed.

Clemson University.

$¥$ Oak Ridge National Laboratory.

\& University of Tennessee.

" Kyoto University.
}

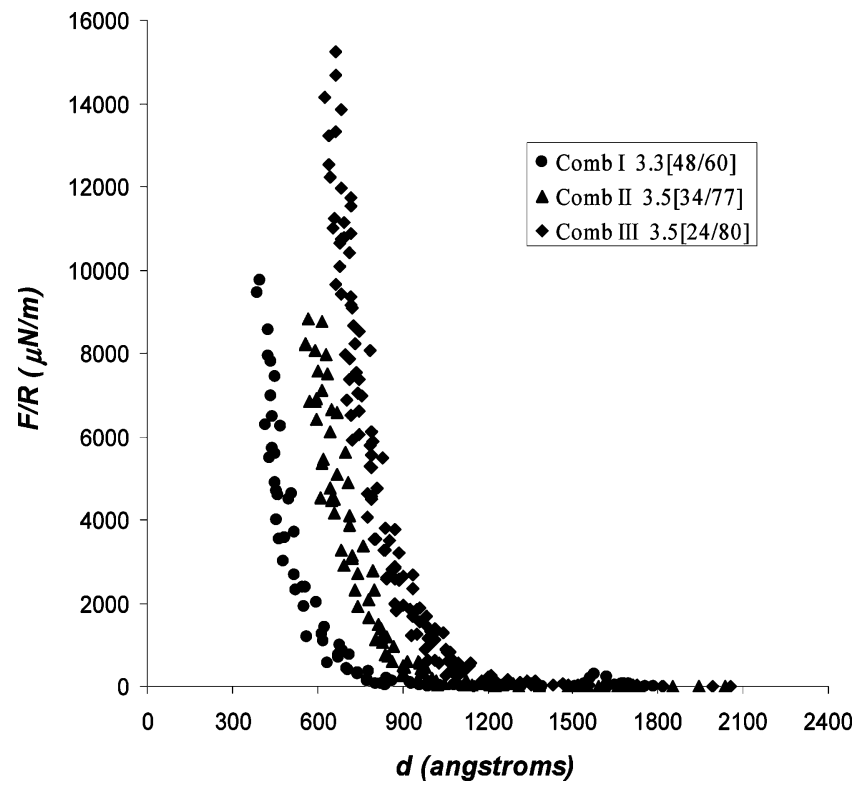

Figure 1. Normal force profiles for PS brushes in MEK made by preferential assembly of PI/PS combs. The range of the profiles reflects the fact that the chains are stretched a few times their free solution radii of gyration.

[24/80], where the number preceding the brackets is the statistical number of branches per comb and the numbers inside the brackets refer to the molecular weights, in $\mathrm{kg} / \mathrm{mol}$, of the PI and PS chains, respectively, that constitute the miktoarm (mixed arm) building block of the comb copolymer. This subunit of the comb can also be thought of as the equivalent diblock copolymer of which the brush is comprised. The synthesis is tailored so that PI blocks cap each end of the comb. ${ }^{6}$ These copolymers were preferentially adsorbed through their PI backbones onto the opposing mica surfaces of the SFA from a nonsolvent for PI, 2-butanone (MEK), creating a PS brush. The "force profiles"-the forces of interaction as a function of surface separation distancewere measured in MEK at $32{ }^{\circ} \mathrm{C}$ after at least $10 \mathrm{~h}$ were allowed for self-assembly and equilibration of the layer. Dry layer thicknesses were measured after the surface forces experiment.

As seen from Figure 1, the normal forces of interaction are monotonically repulsive and the onset of repulsion increases as the size of the PS graft increases and also as the distance between grafting points (along the PI backbone) decreases. The latter point is clearly illustrated by comparing the force profiles for the brushes formed from the 3.5[34/77] and 3.5[24/80] combs. Here, the PS graft size has been increased by $\sim 20$ monomer units, while the molecular weight of the PI chain between the grafts has been substantially reduced by $10.6 \mathrm{~kg} / \mathrm{mol}$, or $\sim 156 \mathrm{PI}$ monomers. This reduction of the PI "length" between grafts causes local crowding within the layer, causing the chains to extend further 
Table 1. Properties of Combs Studied ${ }^{a}$

\begin{tabular}{lcccccc}
\hline copolymer & PI $M_{w}$ & $\mathrm{PS}_{w}$ & $N_{P I}$ & $N_{P S}$ & $\begin{array}{c}\sigma(\mathrm{PS} \\
\text { chains/m }\end{array}$ & $L / R_{g}$ \\
\hline $3.3[48 / 60]$ & 48000 & 60300 & 706 & 580 & $1.93 \times 10^{16}$ & 4.46 \\
$3.5[34 / 77]$ & 34200 & 77300 & 503 & 743 & $2.23 \times 10^{16}$ & 5.02 \\
$3.5[24 / 80]$ & 23600 & 79500 & 347 & 764 & $2.77 \times 10^{16}$ & 6.05
\end{tabular}

$a$ The molecular weight and number of PI (backbone) and PS (grafts) units refers to the constituting miktoarm subunit of the comb. This "equivalent diblock copolymer" is used as the basis for calculating the surface density of grafted PS chains and degree of stretching of the PS grafts, $L / R_{g}$.

from the tethering plane. If we take one-half of the onsets of repulsion as the height of one brush layer and divide this value by the radius of gyration (in free solution) of the PS grafts, ${ }^{7}$ we can obtain a measure of the degree of stretching of the brush. These results, along with the surface density based on the constituting miktoarm subunit of the comb, which is calculated from the dry layer thickness, ${ }^{2,3}$ are reported in Table 1 . There are two important points to make concerning these results: first, preferential adsorption of the comb copolymers results in a slightly higher adsorbed amount compared to what is achieved with diblock copolymers and, second, the degree-of-stretching of the brushes formed from the combs is also slightly higher than what is typically realized with brushes formed from diblock copolymers. ${ }^{3}$ These results are the first indications that connectivity, and therefore, local effects, are important in brushes formed from branched polymer amphiphiles.

To coalesce the force profiles displayed in Figure 1 to a single profile, we reduce the measured forces of interaction, $F / R$, and surface separation distances, $d$, by the scalings for the equilibrium free energy (per unit area) and height, respectively, of a brush comprised of the same PS molecular weight and tethering density:2,3

$$
\begin{gathered}
\boldsymbol{F}=(F / R) / k T b^{1 / v} N \sigma^{(2 v+1) / 2 v} \\
\boldsymbol{d}=\left(d-2 L_{P I}\right) / 2 N b^{1 / v} \sigma^{(1-v) / 2 v}
\end{gathered}
$$

In these equations, the factor $2 L_{P I}$ removes the thickness of the anchoring PI block from the height of the brush, $b$ is the statistical segment size, $\sigma$ is the tethering density based on the equivalent diblock copolymer (the miktoarm subunit of the comb), and $v$ is the parameter that scales radius of gyration with degree of polymerization, $N$. Previous light scattering measurements on PS homopolymers in MEK yielded values of $b=1.88 \AA$ and $v=0.571 .{ }^{7}$ As seen in Figure 2, scaling the SFA results in this fashion gathers the force profiles for the brushes formed from the combs into a single master curve, which demonstrates that the well-known scaling relationships for linear brushes embodied in eqs 1 and 2 are valid for the brushes produced by preferential adsorption of the comb copolymers. This is reasonable given that the Alexander-de Gennes model describes a global balance of the elastic and stretching energies for tethered linear chains, which depends on $N, b$, and $\sigma$ but not local elements, such as how the tethering is achieved. ${ }^{4,5}$

Figure 2 also shows that the master curve resulting from the linear PS brushes formed by selective adsorption of the branched PI/PS copolymers is longer-ranged than the curves created by PS brushes (made from polyvinylpyridine-PS (PVP-PS) diblock copolymers) in toluene (open circles) and in cyclohexane (open squares). The relative positions of these three curves amplify the

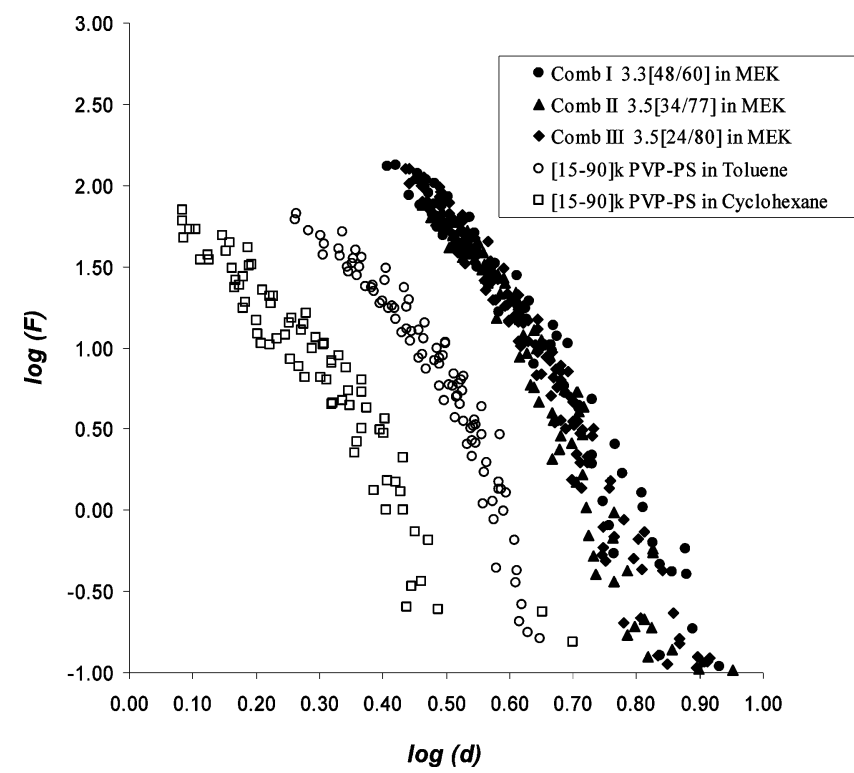

Figure 2. Scaled force profiles for brushes formed from preferentially assembled PI/PS comb copolymers in MEK and a representative PVP-PS diblock in toluene and cyclohexane. The data have been reduced to scale the dependence on molecular weight, tethering density, and segment size. The scaled force profile of the brushes formed from the combs in MEK is longer ranged than that of the brushes formed from diblocks in the good solvent, toluene.

point made previously concerning the $L / R_{g}$ values given in Table 1: the brushes formed from the combs are more strongly stretched than the brushes formed from the diblock copolymers, despite the fact that the solvation power of MEK for PS is intermediate to that of toluene and cyclohexane. The additional stretching that causes extension of the brush beyond what would be expected if the layer were formed from the corresponding linear polymer amphiphile must arise due to the branched structure of the comb and confinement of the chains to the fluid-solid interface. Watanabe, Kilbey, and Tirrell previously showed that the effect of confinement could be accounted for if the proper correlation length for the tethered system was used when evaluating the osmotic free energy of the brush. ${ }^{8}$ To account for the effect of architecture and coalesce the surface forces data for the brushes formed from linear and branched polymer amphiphiles to a single profile, it is necessary to alter further the global description of the tethered layer to include intramolecular effects.

We propose that this can be accomplished through the incorporation of the branching parameter $g_{s}$. This coefficient, defined as the ratio of the measured $z$-average mean square radius of gyration of the branched polymer to that of the corresponding linear polymer of the same molecular weight, is frequently used to characterize the degree of branching within the macromolecule. ${ }^{9}$ It is also related to the ratio of the average concentration of segments within the volumes of the branched and linear chains - as such, $g_{s}$ is a measure of the segment density within the macromolecule. It is well known that branched polymers have higher segment densities compared to the corresponding linear chains and, therefore, increased volume interactions within the molecule. In bulk solutions, this additional monomer-monomer repulsion leads to a decrease in the theta temperature for stars and combs as the number of arms increases. In tethered systems, this effect enhances the ability of the brush to sterically stabilize the surface. ${ }^{3}$ Thus, $g_{s}$ 
accounts for the effect of architecture on the observed difference between reduced force-distance profiles for brushes formed from the linear and branched polymer amphiphiles (Figure 2).

We begin by considering that the structure of the layer results from a balance between the elastic and interaction free energies and by assuming that the brush chains are equally stretched with all chain ends localized at the brush-fluid interface. At this stage, no adjustment is needed for the elastic free energy of the layer-it remains related to the stretching energy for Gaussian chains tethered at $\sigma$ chains per unit area. To modify the osmotic contribution to the overall free energy of the layer, we must first determine whether the osmotic pressure of the brush, $\Pi$, follows the scaling relationship for the concentration dependence of semidilute solutions, $\Pi \sim \xi^{-3} \sim c^{3 v / 3 v-1}$, where $\xi$ is the correlation length (blob size). ${ }^{8}$ Verifying the scaling dependence will allow us to evaluate the osmotic free energy based on the work required to concentrate the chains from their uncompressed state to a particular degree of compression, which mirrors what is done during the surface forces experiment.

An expression for the osmotic free energy of the brush per unit mass of chains, originally derived by Watanabe and Tirrell, is given as eq $3 .^{2}$ In this expression, $m_{o}$ is the monomer mass and $w$ is the mass of chains per unit area, $2 \sigma M_{P S} / N_{A v}$. With this equation, the measured $F / R$, an energy per unit area, can be recast as the reduced osmotic free energy and plotted versus the average concentration of chains between the confining surfaces, $c$, which is inversely related to the surface separation distance. $^{2}$

$f_{o s, b}=\left[\left(m_{o} / N_{A v}\right)^{3 v / 3 v-1} /\left(w k T\left(4 \pi b^{3} / 3\right)^{1 / 3 v-1}\right)\right](F / 2 \pi R)$

As seen in Figure 3, at higher levels of compression, as the segment concentration between the surfaces is increased by squeezing, the curves merge and the osmotic free energy depends on only the average concentration of segments. While this observation confirms the scaling form, the exponent is confirmed by comparing the osmotic free energy of the brush to that of the corresponding homopolymer in the semidilute regime.

The four lines shown in Figure 3 all emerge from expressions for the osmotic free energy of chains and are normalized to the same basis as eq 3 . The mathematical manipulations that give rise to these various lines are delineated in the Appendix-here they are described on a physical basis. Calculation of the osmotic free energy requires an appropriate model for the osmotic pressure-as noted earlier and expressed by eq A1 (in the Appendix), the osmotic free energy is directly related to the work done against osmotic pressure to compress the brushes. The lower line (thin solid line labeled "linear chains in solution") represents the osmotic free energy as a function of concentration for linear chains in solution, as calculated from eq A2. It is based upon the model of des Cloizeaux ${ }^{10}$ for the osmotic pressure of linear chains in semidilute solution. As seen in Figure 3, at moderate levels of compression, the osmotic free energy of the brushes exceeds the osmotic free energy based on linear chains in solution at the same average concentration. The heavy dashed line shown in Figure 3 has the same slope as the thin solid line and represents the limiting behavior at high compression. This line confirms the scaling for the

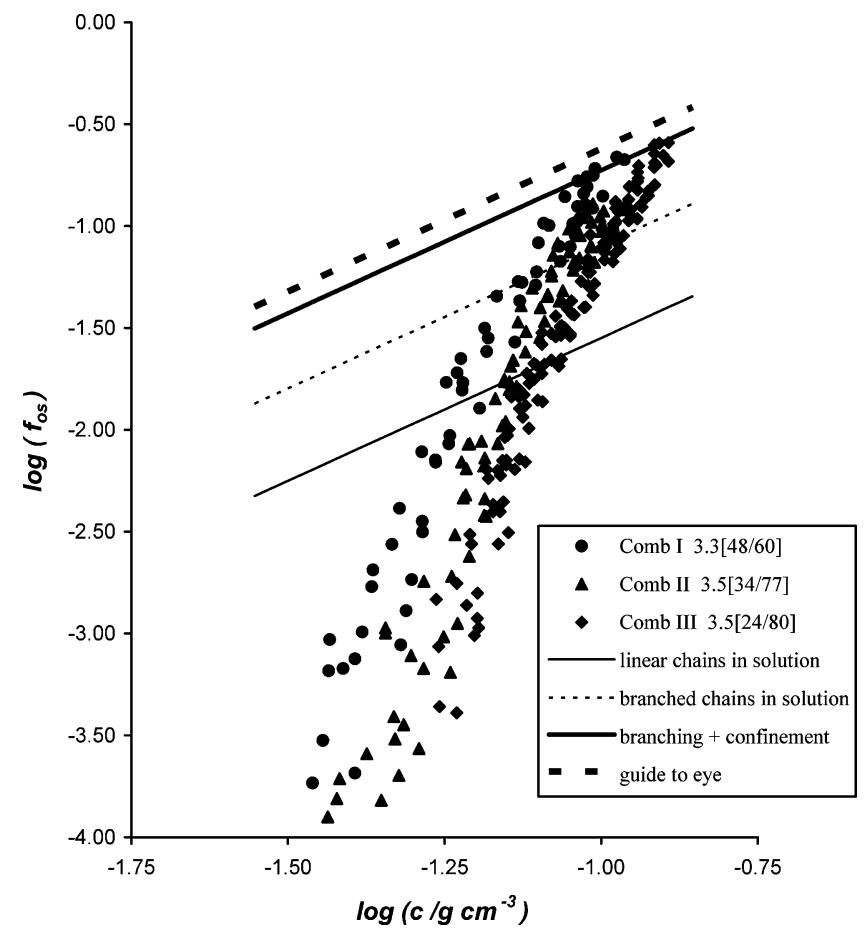

Figure 3. Comparison of the osmotic free energy of the brushes and linear and branched PS homopolymers in MEK (thin solid and thin dashed lines, respectively). The thick solid line represents the osmotic free energy of the layer based on a blob size for the branched layer, and the heavy dashed line is offered as a guide to the eye to reflect the limiting behavior of the system: at high concentration, the curves for the brushes merge, reflecting the fact that the osmotic free energy depends on only the average segment concentration.

concentration dependence, $\Pi \sim \xi^{-3} \sim c^{3 v / 3 v-1}$, but the relative positions of these two lines prove that the osmotic free energy of the brushes made from the combs is underpredicted by a factor of 8.4 if the osmotic pressure is based on a model for linear chains in solution; however, neither the effect of architecture nor confinement has been accounted for.

The effect of branching can be taken into account by using the empirical model proposed by Higo et al. ${ }^{10}$ for the osmotic pressure of regular branched polymers in solution. This model includes the effect of branching through the branching parameter $g_{s}$ and results in the dashed line labeled "branched chains in solution", which was generated using eq A3 and the branching parameter determined from the Berry-Orofino model for normal comb polymers. ${ }^{9}$ Including the effect of branching results in (nearly) a factor of 3 increase in the osmotic free energy compared to linear chains in solution, but the difference between the predicted and measured value remains substantial. Much of this remaining difference should arise due to confinement of the branched chains to the surface.

Fundamentally, this difference between confined and free chains arises because the correlation length of chains in solution and chains confined to the solid-fluid interface are not equal. The models used above calculate the osmotic free energy on the basis of a correlation length (blob size) of the polymers in solution at the overlap concentration, $c^{*} .8$ For coils in solution, the local concentration of segments begins to increase with concentration above $c^{*}$. However, by definition, the chains of the uncompressed brush at equilibrium are already crowded when the layer is assembled-here 
because of confinement to the interface and connectivity of the PS grafts along the backbone. In essence, the local concentration of segments within the brush already exceeds the overlap concentration, and therefore, the correlation length of brushes is smaller than the correlation length of coils in free solution at the same average concentration, $\xi_{b}>\xi_{s}$. Consequently, it is necessary to evaluate the osmotic free energy not on the basis of coils in solution (i.e., not on the basis of $c^{*}$ or $\xi_{s}$ ) but rather on the uncompressed, equilibrium brush. ${ }^{3,8}$

This is done through eq A6, which results in the heavy solid line shown in Figure 3 labeled "branching + confinement". Including the effect of confinement has resulted in a factor of 2.4 increase in the calculated osmotic free energy compared to that predicted for branched chains in solution. These adjustments are nearly enough to produce agreement between the predicted and measured osmotic free energies, and therefore, the osmotic free energy of the layer can be evaluated from eq A6. It is important to point out that the equations that reflect implementation of the various models have no adjustable parameters-values of $K_{\pi}$ and $v$ are known from independent experiments, and the branching parameter and effect of confinement are independently calculated.

Knowing that including the effect of architecture and/ or confinement improves the agreement between the measured and calculated osmotic free energy, we turn our attention to determining whether the combined effects correctly predict the free energy of the brush layer. We do this by recasting the Alexander-de Gennes mean-field model to scale the dependence on tethering density, solvent quality, segment size, and molecular weight (of the brush chains). When recast in this form, ${ }^{3}$ the reduced free energy of the brush as function of the reduced surface separation distance can be written simply as

$$
F^{*}=1+d^{*}
$$

where

$$
\boldsymbol{F}^{*}=\left(\frac{\mathrm{F}}{X}+1+\frac{1}{4 v-1}\right)\left(\frac{4(3 v-1) K_{\pi}}{4 v-1}\right)^{-1 / 4 v}
$$

and

$$
\boldsymbol{d}^{*}=\frac{1}{4 v-1}\left(\frac{4(3 v-1) K_{\pi}}{4 v-1}\right)^{-1}\left(\frac{4 \pi}{3 \beta}\right)^{2(v-1) /(3 v-1)} \boldsymbol{d}^{4 v /(3 v-1)}
$$

and the constant $X$ is defined as

$$
X=4 \pi[(4 v-1) / 4]^{1 / 4 v}\left[(3 v-1) K_{\pi}\right]^{(4 v-1) / 4 v}(4 \pi / 3 \beta)^{1 / 2 v}
$$

This model, which relies on three parameters, $v, K_{\pi}$, and $\beta$, has been used successfully to coalesce the forcedistance profiles of PS brushes in good and near-theta solvents to a single master curve, forming a "universal profile". ${ }^{3}$ As detailed in the Appendix, the constant $\beta$ appears because we have replaced $c^{*}$ by $\beta c^{*}$ in the models for the osmotic pressure as a function of concentration, ${ }^{10}$ thereby allowing us to incorporate, in a single multiplicative parameter, the effect of architec-

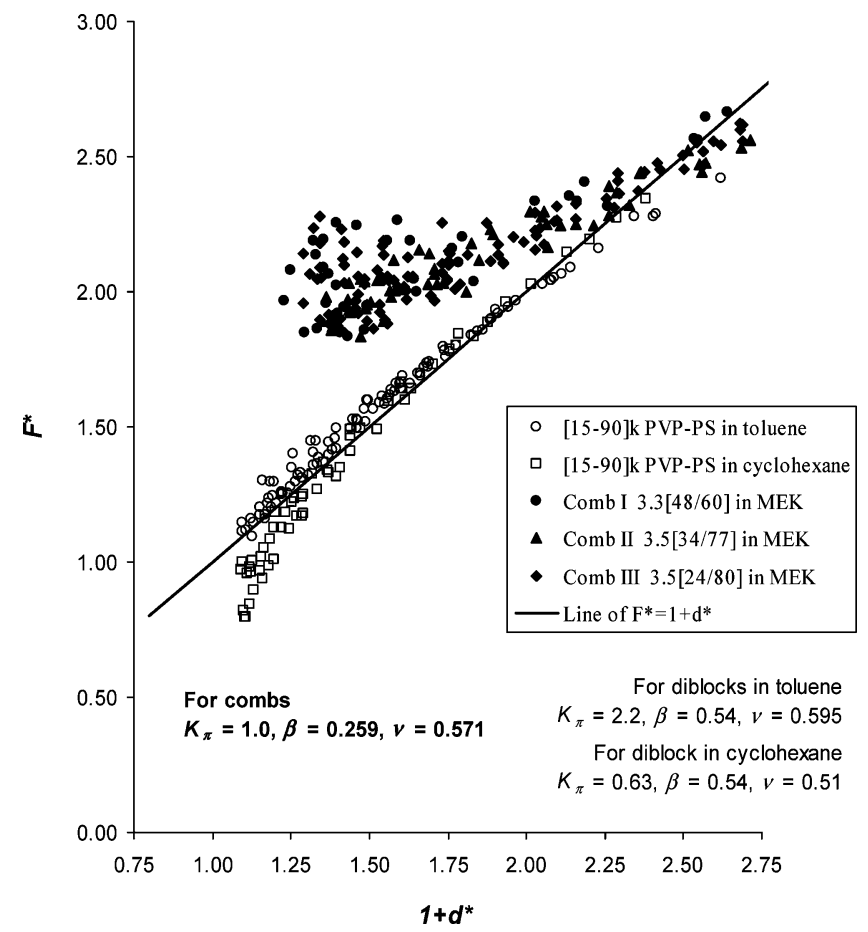

Figure 4. Universal profile for brushes. By including the effect of confinement and branching, the force profiles for the brushes formed from the combs nearly coalesce with those of PS brushes formed from linear PVP-PS copolymers. The remaining discrepancy is attributed to the rubbery PI blocks that anchor the combs to the surface.

ture and confinement on the osmotic free energy of brushes. This also leads to a change in the numerical prefactor on the elastic stretching energy term. ${ }^{2}$

Given the analyses of the effect of architecture and confinement on the osmotic free energy of the brush, it is straightforward to determine a numerical value for $\beta$ from eq A6. This yields a value of $\beta=0.259$, which along with values of $v$ and $K_{\pi}^{7,11}$ and the experimentally determined reduced force and distance data $(\boldsymbol{F}$ and $\boldsymbol{d})$, leaves no adjustable parameters in the model expressed by eq 4 . The data for the brushes produced from the combs recast according to eq 4 are plotted in Figure 4, along with a pair of representative results from a PS brush in toluene and near-theta cyclohexane formed from a preferentially adsorbed PVP-PS diblock copolymer.

As seen in Figure 4, the reduced force-distance profiles from the PI/PS combs tend toward, but do not overlay, the universal curve obtained for PS brushes formed from PVP-PS diblock copolymers, despite including the effect of branching and confinement on the free energy of the layer. However, neglecting either one of these effects would shift the scaled data off of the plot area. Thus, the discrepancy between the data and model reveals that a relatively small structural effect has not been captured. We propose that the difference is due to the rubbery nature of the PI anchor block and speculate that those hydrophobic blocks do not lay flat along the hydrophilic mica surfaces because of the difference in surface energy or/and tendency for dewetting (aided by the mobility of the PI blocks). Both of these would result in a nonuniform anchoring layer and manifest as an additional repulsion that shifts the force profiles to greater distances. This idea is depicted in Figure 5. In summary, we have shown that local effects such as connectivity can have significant impact on the struc- 


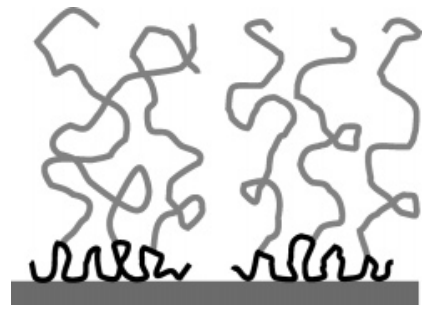

Figure 5. Sketch capturing the proposed structure of the brush formed from preferentially assembled PI/PS comb copolymers in MEK. The hydrophobic PI blocks may not lay flat (anchor strongly) along the mica surfaces.

ture of polymer brushes. From a practical point of view, this suggests that architecture becomes another important tool for tailoring the interfacial properties of brushbearing surfaces. By properly accounting for the effect of branching through incorporation of the branching parameter, it appears possible to develop a universal profile that adequately describes, by scaling the dependence on solvent quality, tethering density, segment size, molecular weight, and branching, the structure of these linear brushes in good solvents. This model contains no adjustable parameters; however, it should be noted that this strictly applies for brushes in good solvents, as in poorer solvents, tethering the chains to the interface produces an additional monomer-monomer repulsion that manifests as a change in $v^{3}$

\section{Appendix}

Here we describe in detail the manipulations that give rise to the series of lines in Figure 3. As explained in the body of this Note, these lines describe the osmotic free energy of the homopolymer system as a function of average concentration. The magnitude of the osmotic free energy of the system changes as the effects of chain architecture and confinement are included. As explained by Watanabe and Tirrell, ${ }^{2}$ the osmotic free energy of the brush layer, $f_{o s}$, as a function of concentration can be evaluated as the work per area done against the osmotic pressure to bring the brushes from separated and noninteracting $(x=\infty)$ to separation distance $d$ :

$$
f_{o s}=-K \int_{x=\infty}^{x=d} \Pi[c(x)] \mathrm{d} x
$$

In this form, the constant $K$ is comprised as the same collection of terms in the square brackets of eq 3 . This normalization allows the osmotic free energy calculated from SFA data (i.e., eq 3) to be directly compared with that predicted from eq $\mathrm{A}$.

The first comparison of the osmotic free energy of the brushes made is with linear PS homopolymers in solution at the same average concentration, $c$. To do this, the empirical relationship (of des Cloizeaux form) describing the osmotic pressure of a semidilute polymer solution, $\Pi M / c R T=K_{\pi}\left(c / c^{*}\right)^{1 / 3 v-1},{ }^{10}$ is inserted into eq A1 and integrated. This yields

$$
f_{o s, s}=(3 v-1) K_{\pi} c^{1 / 3 v-1}
$$

where $f_{o s, s}$ is the osmotic free energy of linear chains in solution. The additional subscript " $s$ " is added to distinguish the fact that this osmotic free energy, by virtue of being based on the des Cloizeaux model, ${ }^{10}$ is based upon the correlation length for free chains in solution, $\xi_{s} \sim\left(c / c^{*}\right)^{-v /(3 v-1)}$. As can be seen in Figure 3, when the layers are concentrated by compressing the brushes against one another, the osmotic free energy rapidly exceeds the osmotic free energy predicted by eq A2 (represented by the thin solid line and labeled "linear chains in solution") when the experimentally determined parameters of $v=0.571$ and $K_{\pi}=1.0$ for PS in MEK $^{11}$ are used.

The effect of branching can be incorporated using the empirical model of Higo et al. ${ }^{10}$ who showed for homopolymer stars and combs that if $v$ for the branched and linear polymers is the same and $\xi$ for branched and linear polymers has the same concentration dependence, then an appropriate model for the osmotic pressure of branched polymers is $\Pi M / c R T=g_{s}^{-3 / 2(3 v-1)} K_{\pi}\left(c / c^{*}\right)^{1 / 3 v-1}$, where the prefactor $g_{s}{ }^{-3 / 2(3 v-1)}$ comes from basing the overlap concentration on the radius of gyration of the branched polymer. ${ }^{10}$ Inserting this expression for the osmotic pressure into eq $\mathrm{A} 1$ and carrying out the integration yields

$$
f_{o s, s b}=(3 v-1) K_{\pi} g_{s}^{-3 / 2(3 v-1)} c^{1 / 3 v-1}
$$

This expression for the osmotic free energy is analogous to that of eq A2 in form and also is based upon the correlation length of chains in solution. The additional letter " $b$ " appended to the subscript used in eq A3 clarifies that this osmotic free energy includes the effect of branching based on branched chains in solution. To determine $g_{s}$, we use the Berry-Orofino equation for normal comb polymers ${ }^{9}$ and calculate $g_{s}=0.61$ for the PI/PS combs investigated. This value of $g_{s}$ and the aforementioned values of $K_{\pi}$ and $v$ produce the dashed line shown in Figure 3 that is labeled "branched chains in solution". As noted in the body of the Note, including the effect of branching results in a slightly less than 3 -fold increase in the calculated osmotic free energy:

$$
f_{o s, s b} / f_{o s, s}=g_{s}{ }^{-3 / 2(3 v-1)}=2.84 \text { for } v=0.571
$$

However, the osmotic free energy of the brush formed from the comb copolymers remains underpredicted by a factor of approximately 3 .

The next line in Figure 3, which is labeled "confinement + branching", comes about by including the effect of confinement and branching in calculating the osmotic free energy. Our previous papers on the scaling analysis of linear polymer brushes describe how the effect of confinement is taken into account. ${ }^{3,8}$ As described earlier, because the chains of a brush are already crowded in the uncompressed state, the correlation length of brushes is smaller than the correlation length of the corresponding polymer solution at the same average concentration. Consequently, it is necessary to evaluate the osmotic free energy of the brush, $f_{o s, b}$, based not on coils in solution (i.e., based not on $c^{*}$ or $\xi_{s}$ ), but rather on the uncompressed, equilibrium brush. ${ }^{3,8}$

A scaling model that allows $f_{o s, b}$ of the brush to be explicitly determined was developed by Watanabe et al. ${ }^{8}$ and the relevant points are summarized here. In the model, the correlation length used to determine the osmotic pressure within the brush layer, $\Pi_{b}$, was based on the equilibrium concentration of segments in the layer, $c_{e q}, \xi_{b} \sim\left(c / c_{e q}\right)^{-v / 3 v-1}$ and $\Pi_{b} \sim \xi_{b}{ }^{-3}$. The osmotic free energy of the brush based on $\xi_{b}$ can then be calculated as the work done against osmotic pressure to compress the brush. When all numeric prefactors are included and $c_{e q}$ determined self-consistently, an explicit expression for $f_{o s, b}$ as a function of concentration is 
produced. Both $f_{o s, b}$ and $f_{o s, s}$ have the same concentration dependence, differing only in magnitude, and the ratio expresses the degree to which the osmotic free energy of the layer is underpredicted if based upon $\xi_{s}$ rather than $\xi_{b}: 2,3$

$$
\frac{f_{o s, b}}{f_{o s, s}}=\left(\frac{3 \sqrt{3}}{2 \sqrt{2}}\right)^{1 /(3 v-1)}=Q
$$

Therefore, for the branched brushes studied here we can write

$$
\begin{array}{r}
f_{o s, b b}=Q f_{o s, s b}= \\
\left(\frac{3 \sqrt{3}}{2 \sqrt{2}}\right)^{1 /(3 v-1)} g_{s}^{-3 / 2(3 v-1)}(3 v-1) K_{\pi} c^{1 / 3 v-1}= \\
(3 v-1) K_{\pi}\left(\frac{c}{\beta}\right)^{1 / 3 v-1}
\end{array}
$$

Here the parameter $\beta\left(=\left\{2 g_{s} / 3\right\}^{3 / 2}\right)$ collects the effects of branching and confinement into a single multiplicative parameter that operates on $c^{*}$ in the model for the osmotic pressure-in essence, rescaling the correlation length for the brush formed from the branched copolymers. This expression for the osmotic free energy of the brush formed from the branched polymers has no adjustable parameters and, as seen in Figure 3, adequately predicts the osmotic free energy of the compressed PS brushes made from preferentially assembled PI/PS comb copolymers.
Acknowledgment. This work was supported by the National Science Foundation through the Chemistry Projects Program (Award No. 0304807) and the Interfacial Transport and Separations Program (Award No. CTS-0129613). Support from the Division of Materials Sciences and Engineering, U.S. Department of Energy, under contract DE-AC05-00OR22725 with UT-Battelle, LLC, is also gratefully acknowledged.

\section{References and Notes}

(1) Halperin, A.; Tirrell, M.; Lodge, T. P. Adv. Polym. Sci. 1992, 100, 31-71.

(2) Watanabe, H.; Tirrell, M. Macromolecules 1993, 26, 64556466.

(3) Kilbey, S. M., II; Watanabe, H.; Tirrell, M. Macromolecules 2001, 34, 5249-5259.

(4) Alexander, S. J. J. Phys. (Paris) 1977, 38, 983-987.

(5) de Gennes, P. G. Macromolecules 1980, 13, 1069-1075.

(6) Uhrig, D.; Mays, J. W. Macromolecules 2002, 35, 71827190.

(7) Lewis, M. E.; Nan, S.; Yunan, W.; Li, J.; Mays, J. W. Nadjichristidis, N. Macromolecules 1991, 24, 6686-6689. Horita, K.; Abe, F.; Einaga, Y.; Yamakawa, H. Macromolecules 1993, 26, 5067-5072.

(8) Watanabe, H.; Kilbey, S. M., II; Tirrell, M. Macromolecules 2000, 33, 9146-9151.

(9) Berry, G. C.; Orofino, T. A. J. Chem. Phys. 1964, 40, 16141621.

(10) Higo, Y.; Ueno, N.; Noda, I. Polym. J. 1983, 15, 367-375.

(11) Bawn, C. E. H.; Freeman, R. F. J.; Kamaliddin, A. R. Trans. Faraday Soc. 1950, 46, 862-872.

MA049021U 